\title{
Comparaison de l'efficacité d'une technique de lutte chimique et d'une technique de lutte biologique pour la protection de la culture de la tomate contre l'acarien tisserand Tetranychus urticae (Acari: Tetranychidae)
}

\author{
T Hance 1, G Van Impe 1, P Lebrun 1, P Nihoul 1, F Benoit 2, N Ceusterman 2 \\ 1 Institut pour l'encouragement de la recherche scientifique dans l'industrie et l'agriculture (IRSIA), centre d'acarologie, \\ unité d'écologie et de biogéographie, Place Croix du Sud, 5, B-1348 Louvain-la-Neuve; \\ 2 Proefstation voor de Groenteteelt, B-2580 Sint-Katelijne-Waver, Belgique
}

(Reçu le 22 février 1991; accepté le 1er juillet 1991)

\begin{abstract}
Résumé - Deux techniques visant à la protection de la culture de la tomate en serre contre l'acarien tisserand ( $T e$ tranychus urticae) ont été comparées. Pour la première, une introduction préalable du ravageur sur la culture a été réalisée, suivie de l'introduction de 2 prédateurs, Amblyseius andersoni et Phytoseiulus persimilis. Ceci dans le but d'assurer une colonisation réussie de la culture par les prédateurs durant toute la saison. La seconde technique con-siste en l'utilisation d'un acaricide conventionnel. Les rendements en poids des tomates produites, les calibres des fruits et le revenu brut ont été calculés dans les 2 cas. Bien que les dégâts foliaires aient été plus importants dans le cas de la lutte biologique, aucune différence significative n'a été observée entre les 2 traitements relativement à l'importance de la production ou au calibre des fruits. Par contre, en ce qui concerne le revenu, on observe un excédent positif en faveur de la lutte biologique.
\end{abstract}

contrôle biologique / Tetranychus urticae / Lycopersicon esculentum = tomate / Phytoseiulus persimilis / Amblyseius andersoni / introduction de prédateurs / index de dégâts / Pest in First

Summary - Comparison of biological and chemical control methods for tomato crop protection against the two-spotted spider mite Tetramychus urticae (Acari: Tetranychidae). Two methods to control the two-spotted spider mite (Tetranychus urticae) on glasshouse tomatoes were compared. In the first method, an initial introduction of the pest was followed by the introduction of 2 predators, Amblyseius andersoni and Phytoseiulus persimilis. These predators differ in their demographic properties and polyphagy and should exert a complementary action on the pest population. The aim of this "pest in first" technique was to ensure a successful colonization of the crop by the predators throughout the season. In order to avoid any interference, biological control was applied against the other pest problems. The second method consisted of a chemical pest control. Each week, both crops were observed and the evolution of leaf damage was recorded by means of a visual index (figs 1,2). Moreover, yield, fruit size and gross income were recorded in both cases (tables II, III, IV). Leaf damage was greater in the biological control greenhouse, and this led to further introduction of predators. But at the end of the season, no significant differences in yield and fruit size were observed between treatments. Nevertheless, gross income was greater in the biological control greenhouse. This experiment shows that biological control may be a feasible alternative in tomato glasshouse crops.

biological control / Tetranychus urticae / Lycopersicon esculentum = tomato $/$ Phytoseiulus persimilis $/$ Amblyseius andersoni / predator introduction / damage index / Pest in first 


\section{INTRODUCTION}

La technique de l'introduction préalable du ravageur (IPR, aussi appelé Pest in first) a été mise au point et développée en Grande-Bretagne par Hussey et Scopes (1985), pour protéger les cultures de concombres (Cucumis sativus $L$ ) et de tomates (Lycopersicon esculentum Miller) en serre contre l'acarien tisserand (Tetranychus urticae Koch).

Le principe de cette technique repose sur l'introduction volontaire, en début de culture, du phytophage et de son prédateur en proportions définies, afin d'assurer la présence permanente du prédateur dans la serre, et de contrecarrer ainsi toute infestation ultérieure de la culture par l'acarien phytophage. Ces infestations découlent fréquemment de l'immigration de quelques individus par les sytèmes d'aération ou, au printemps, de la levée de la diapause hivernale des tétranyques présents dans la serre à l'automne précédent. Les dégâts occasionnés par ces phytophages sont importants et le préjudice économique qui en résulte peut être grave.

Le prédateur le plus couramment utilisé contre $T$ urticae est un autre acarien Phytoseiulus persimilis Athias-Henriot (Phytoseiidae), dont la réponse numérique au développement des populations de la proie est très rapide (Hance et Pasleau, 1987; Hance, 1988). II détruit rapidement sa proie mais, comme il s'agit d'un prédateur spécifique, il se trouve à bref délai dans l'impossibilité de se nourrir. II manifeste dans ces conditions une forte tendance à la dispersion (Bernstein, 1985); cependant, lorsque celle-ci n'est pas possible, il meurt d'inanition ou se livre au cannibalisme.

En pratique, ce prédateur est souvent utilisé par les horticulteurs selon le même principe qu'un agent acaricide. À l'apparition des premiers symptômes d'attaque par T urticae, une introduction de prédateurs adultes est opérée sur les plantes infestées. S'il est en nombre suffisant, $P$ persimilis détruit rapidement l'acarien phytophage, puis disparaît à son tour. Dès lors, si une nouvelle infestation se produit ultérieurement, l'horticulteur est contraint d'effectuer un nouveau lâcher de prédateurs.

Amblyseius andersoni (Chant) diffère fondamentalement de $P$ persimilis par ses caractéristiques démographiques et sa réponse numérique plus lente que celle de $P$ persimilis par rapport à la croissance des populations de la proie (Amano et Chant, 1977).
C'est un prédateur polyphage, qui, bien que se nourrissant de $T$ urticae, peut également effectuer la totalité de son développement grâce à une autre nourriture, comme le pollen, et exprimer une fécondité normale sur ce substrat. C'est sans doute cette caractéristique qui explique la réponse lente de ce prédateur à l'accroissement des effectifs de sa proie. En verger de pêchers (Putman et Herne, 1966) de pommiers (Rambier et Van de Vrie, 1974; Gambaro, 1983), en verger et en viticulture (Baillod et al, 1982), cette espèce est reconnue comme un prédateur important de Panonychus ulmi (Koch) et de T urticae. Cependant, des expériences réalisées en conditions contrôlées en laboratoire ont montré que lorsque la densité de sa proie devient trop élevée, $\boldsymbol{A}$ andersoni est incapable de contrôler la multiplication des effectifs et d'empêcher l'épuisement de la plante hôte (Hance et al, 1991). Introduite seule en serre, cette espèce ne pourrait protéger la culture durablement.

Par sa polyphagie, $A$ andersoni offre néanmoins l'avantage de pouvoir être introduit en serre en début de culture, et de pouvoir s'y maintenir en l'absence de l'acarien phytophage à condition de pouvoir bénéficier d'une source de nourriture alternative. En présence de pollen, il pourrait ainsi se multiplier et contrecarrer une éventuelle infestation par $T$ urticae. De telles observations ont déjà été réalisées pour une espèce proche, Neoseiulus cucumeris (Oudemans) capable de se multiplier en serre uniquement grâce à la présence de pollen de poivron (Caspicum annuum L) (Van Rijn et Sabelis, 1990).

Dans le cadre de ce travail, les 2 prédateurs présentés ont été choisis en raison de leur propriétés biologiques complémentaires. En effet, lorsque $P$ persimilis est utilisé seul en culture de tomates, en fin de saison des populations résiduelles de $T$ urticae se maintiennent en serre et sont à tout moment une source potentielle de réinfestation de la culture qui peut compromettre l'équilibre proie-prédateur (Nihoul et Van Impe, 1991). De plus, ces populations sont localisées dans le bas des plantes, alors que $P$ persimilis se situe essentiellement sur la partie supérieure des plantes. A andersoni pourrait agir efficacement sur ces populations résiduelles qui survivent à basse densité.

L'objectif de cette étude est de comparer l'évolution des dégâts foliaires sous 2 programmes de protection, l'un utilisant les 2 prédateurs mentionés ci-dessus après introduction du ravageur et de pollen, l'autre faisant appel à l'utilisation d'un acaricide classique. Une évaluation des rende- 
ments et des coûts associés aux 2 méthodes sont des critères choisis en raison de leur importance pour les horticulteurs.

\section{MATÉRIEL ET MÉTHODES}

Les essais ont été réalisés dans une serre de $140 \mathrm{~m}^{2}$, séparée en 2 compartiments. La fertilisation du sol a été assurée avec $6 \mathrm{~kg}$ de patenkali, $3 \mathrm{~kg}$ d'engrais composés $\mathrm{N}, \mathrm{P}, \mathrm{K}(12-12-17)$ et $2 \mathrm{~kg}$ de $\mathrm{Mg}$ pour $100 \mathrm{~m}^{2}$.

Trois cultivars de tomates ont été utilisés: Caruso, Fernova et Vedettos, à raison de 3 lignes pour les cultivars Caruso et Vedettos et de 2 lignes pour Fernova. Les graines ont été semées le 21 mars, les plants repiqués en pots de tourbe le 5 avril, et installés dans la serre le 27 avril 1989. La distance entre plants était de $60 \mathrm{~cm}$ dans la ligne, avec 8 lignes sur la largeur de la serre $(6,4 \mathrm{~m})$. Dans le cadre de cette expérimentation, la culture a été volontairement limitée à 9 bouquets (étages floraux). La récolte des tomates a débuté le 26 juin et s'est terminée le 22 août 1989. Pour chaque cultivar, la production quotidienne en tomates a été évaluée par plant, pour 20 plants répartis sur 2 lignes distinctes. De plus, les tomates ont été triées en 3 classes de calibre, à savoir : $<57 \mathrm{~mm}, 57-67 \mathrm{~mm}$, et $>67 \mathrm{~mm}$. Pour chaque classe de calibre, les prix pratiqués tous les $2 \mathrm{j}$ à la vente à la criée de Sint-KatelijneWaver ont été notés, ce qui a permis de calculer le revenu brut de la récolte. Un test de $\chi^{2}$ a été utilisé pour comparer les différences entre compartiments sur le plan des rendements, des calibres et des revenus de la culture.

\section{Lutte chimique}

Dans le compartiment A, pour lutter contre l'acarien tisserand, une décision a été prise de traiter chaque fois que 20 plants présentaient un indice foliaire de dégâts (décrit ci-dessous) de 1,2. Ce critère est en fait supérieur à celui utilisé dans la pratique par la plupart des horticulteurs qui traitent dès l'apparition des premiers symptômes. C'est l'acaricide hexythiazox (Nissorum ${ }^{\left({ }^{\circledR}\right.}$ (BASF) $10 \%$ ), à raison de $5 \mathrm{~g}$ de $\mathrm{ma} / 100 \mathrm{~m}^{2}$ dans $10 \mathrm{I}$ d'eau qui a été choisi pour ces traitements.

En outre, 2 pulvérisations contre l'oïdium (Leveillula taurica (LEV)) furent effectuées ( 21 et 28 juillet) avec du bitertanol (Baycor $\mathrm{EC}^{\circledR}, 30 \%$ ) à la dose de $4,5 \mathrm{~g}$ de $\mathrm{ma} / 100 \mathrm{~m}^{2}$ dans 10 I d'eau et, le 10 juillet, un traitement à base de buprofézine (Applaud ${ }^{\circledR}, 25 \%$ ) contre la mouche blanche Trialeurodes vaporariorum (Westwood) a été réalisé à une dose de $0,75 \mathrm{~g}$ de $\mathrm{ma} / 100$ $\mathrm{m}^{2}$ dans 10 / d'eau.

Dans le compartiment $B$, le seul traitement chimique appliqué était également une pulvérisation de bitertanol à la dose de $4,5 \mathrm{~g}$ de $\mathrm{ma} / 100 \mathrm{~m}^{2}$ dans $10 \mathrm{I}$ d'eau dans le but de circonscrire une infection d'oïdium. Selon Hassan et Oomen (1985), ce fongicide ne possède aucun effet secondaire contre $P$ persimilis.

\section{Lutte biologique}

$A$ andersoni a été introduit le 19 mai dans le compartiment $B$, à raison de 2 femelles adultes par plant tous les 2 plants. Ces acariens provenaient d'un élevage réalisé au laboratoire. Les plants de tomates ont ensuite été saupoudrés de pollen dans le but d'assurer une possibilité d'alimentation durable pour ces prédateurs. Le pollen était composé d'un mélange d'espèces dans les proportions suivantes : $65,2 \%$ de Rosacées (pruniers et cerisier), $10 \%$ de Malus sylvestris Miller, $9,4 \%$ de Taraxacum sp, 4,3\% de Renonculacées, $4,14 \%$ de Salix sp, $3,98 \%$ de Crucifères, $1,1 \%$ de Plantago sp, et moins de $1 \%$ de Aesculus sp, de Graminées et d'Ombellifères. Cette analyse a été réalisée par le Centre apicole de recherches et d'information à Louvain-la-Neuve.

Lorsque les tomates ont atteint le stade de 10 feuilles, 10 femelles du $T$ urticae (adultes et âgées d'1 j) ont été introduites par plant sur les 2 premiers, les 2 derniers plants ainsi que sur les 2 plants centraux de chaque ligne. Ces femelles appartenaient à la souche White Eye (yeux dépigmentés) élevée au laboratoire. Cette mutation récessive permet de distinguer aisément les acariens volontairement introduits de ceux provenant de l'extérieur car les tétranyques sauvages sont caractérisés par des yeux rouges. La méthode d'obtention de femelles de même âge est décrite par Van Impe (1985). Trois jours plus tard, 4 femelles adultes de $P$ permilis ont été placées sur les mêmes plants préalablement infestés par $T$ urticae. Ce prédateur provenait d'élevages commerciaux (Spidex ${ }^{\circledR}$, Koppert, Wageningen, Pays-Bas).

Pour éviter d'interférer avec la mise en place du contrôle biologique des acariens, 2 lâchers d'Encarsia formosa Gahan (En-strip ${ }^{\circledR}$, Koppert) ont été effectués (10 mai et 27 juin 1989), et 6 pièges jaunes englués ont été installés (19 mai 1989) pour lutter contre la mouche blanche (Trialeurodes vaporariorum). Un lâcher de Neoseiulus cucumeris (Oudemans) (Tripex$C^{\circledR}$, Koppert) a été réalisé le 10 mai 1989 pour lutter contre les thrips (Frankliniella occidentalis (Pergande)) et, simultanément, Diglyphus isaea (Walker) et Dacnusa sibirica Telenga (Minex ${ }^{\circledR}$, Koppert) furent introduits pour éliminer la mouche mineuse des feuilles (Liriomyza trifolii Burgess).

\section{Évaluation des dégâts foliaires}

Chaque semaine, la culture a été examinée (compartiments $A$ et $B$ ), et une valeur d'indice de dommage foliaire (IDF, Leaf damage index) a été déterminée visuellement sur les 8 feuilles (minimum 56 folioles) du tiers supérieur de chaque plant. Cet indice comprend 5 niveaux (de 1 à 5), selon l'intensité des symptômes observés (fig 1). Cet index se distingue de celui de Hussey et Scopes (1985) par le fait qu'il est appliqué à la foliole et non pas à la feuille entière du plant de tomate. En effet, ces feuilles montrent une grande variabilité s'agissant de leur forme et donc de leur surface, du nombre de folioles voire de sous-folioles. 

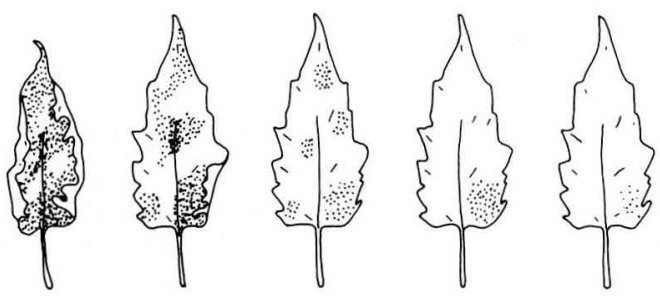

Fig 1. Description des 5 niveaux de l'échelle visuelle d'estimation des dégâts pour l'indice de dommage foliaire au niveau des folioles (IDF). De droite à gauche :

- degré 1 : apparition des premières piqûres de tétranyques, - degré 2 : apparition d'une plage de piqûres résultant de l'extension de la population de tétranyques depuis le point d'infection,

- degré 3 : apparition de nouvelles plages de piqures.

- degré 4 : le foliole est fortement attaqué mais présente encore des zones non piquées, apparition des premières nécroses,

- degré 5 : le foliole ne présente plus de zones non piquées, nécrose totale et dessèchement.

L'observation des folioles diminue donc la variabilité des résultats. L'estimation des dégâts occasionnés à chaque plant a été obtenue par l'intégration des valeurs de I'IDF correspondant à chaque foliole examiné, et le niveau de dégâts moyen fut évalué pour l'ensemble des 15 plants qui comprenait chaque ligne de chaque compartiment. Une estimation des densités de tétranyques pour chaque degré de dégâts est donnée par Maréchal (1990).

En fin de culture, le 16 août, 40 folioles de niveau 3 (IDF) ont été prélevés uniformément dans le compartiment $B$. Les folioles ont été immédiatement congelés $\left(-20^{\circ} \mathrm{C}\right)$ afin de faciliter ensuite l'observation à la loupe binoculaire. Les acariens présents ont été comptés sur chaque foliole en distinguant les espèces et les stades. Cependant, les 2 espèces, $A$ andersoni et $N$ cucumeris n'ont pas été distinguées. De même, les œufs des différentes espèces de prédateurs n'ont pas pu être différenciés entre eux.

\section{RÉSULTATS}

\section{Dégâts foliaires dans le cas de la lutte chimique}

Pendant le mois de juin, dans le compartiment $A$, l'observation des dégâts a révélé quelques foyers localisés provoqués par la souche sauvage (yeux rouges) de $T$ urticae. Ensuite, les dégâts se sont également amplifiés le long des parois de la serre. Dès lors, 3 traitements successifs ont dû être réalisés (les 7,21 et 28 juillet), avec l'acaricide Nissorun (BASF) (10\% hexythiazox). À la suite de ces pulvérisations, I'IDF n'a pas dépassé le niveau 2 (fig 2).

\section{Dégâts foliaires dans le cas de la lutte biologique}

Depuis l'introduction du couple $T$ urticae - $P$ persimilis par la technique IPR et d'A andersoni jusqu'à la première semaine du mois de juillet, I'IDF moyen par plant et par ligne n'a jamais dépassé la valeur 1,5 , reflètant la présence de quelques foyers d'infection (fig 2). Par la suite, une infestation plus importante par $T$ urticae a débuté sous les fenêtres d'aération et le long des parois latérales de la serre. Il s'agissait bien d'un apport extérieur car les tétranyques observés présentaient tous des yeux pigmentés. Étant donné que le caractère "White eye" est récessif, les Tétranyques avec les yeux pigmentés ont rapidement supplanté les formes décolorées qui ont complètement disparu après quelques semaines.

L'infestation s'est développée essentiellement sur les 2 lignes contiguës aux parois, comprenant les cultivars Caruso et Vedettos. À la suite des conditions climatiques exceptionnelles du

A)

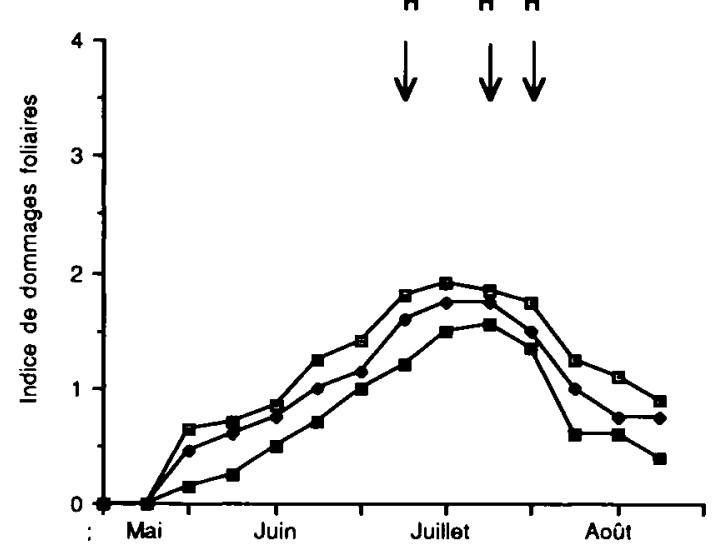

B)

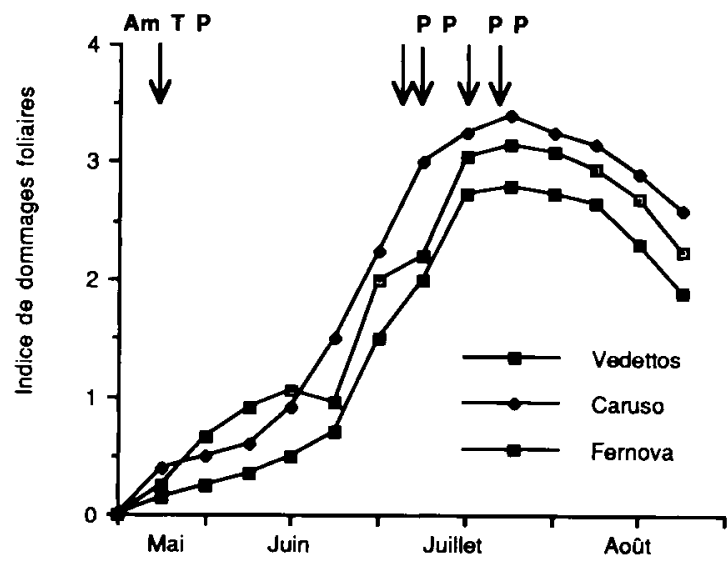

Fig 2. Évolution de l'indice de dommage foliaire de la culture, en fonction du type de protection adoptée vis-à-vis de Tetranychus unticae. A) lutte chimique; B) lutte biologique; Am : introduction de Amblyseius andersoni et de pollen; $\mathrm{H}$ : traitement acaricide; $T$ : introduction du Tetranychus urticae; $\mathrm{P}$ : lâcher du Phytoseiulus persimilis. 
mois de juillet 1989 durant lequel la température extérieure dépassait régulièrement $30^{\circ} \mathrm{C}$, la multiplication de $T$ urticae a été très rapide, et a nécessité 3 apports supplémentaires de 1000 prédateurs ( $P$ persimilis) qui furent répartis uniformément dans le compartiment $B$ les 7,11 , et 18 juillet. Un dernier lâcher eut lieu le 25 juillet, mais uniquement sur les lignes extérieures de ce compartiment. Le 16 août, un examen effectué à la loupe boniculaire a révélé l'abondance des prédateurs présents (en moyenne 4,5 prédateurs par foliole, tous stades et espèces confondus), et la densité très faible de $T$ urticae (tableau I), notamment en comparaison avec les données de Maréchal (1990).

Pendant le mois de juillet, l'évolution des dégâts sur les plants des 2 lignes extérieures (Caruso et Vedettos) a été rapide, atteignant un IDF par ligne de 2,5 le 11 juillet et de 3,5 le 27 juillet. Cette valeur s'est maintenue pour diminuer ( 1,8 pour Fernova, 2,1 pour Vedettos et 2,6 pour Caruso) au mois d'août avec l'apparition de jeunes feuilles non attaquées.

\section{Comparaison des rendements}

Les rendements ne diffèrent pas significativement pour les 3 cultivars $(P>0,05)$. Pour Caruso
Tableau I. Nombre de Tetranychus urticae, de Phytoseiulus persimilis et de Amblyseius sp (A sp) par foliole le 16 août. $m$ : moyenne, $s$ : écart type.

\begin{tabular}{|c|c|c|c|c|c|c|}
\hline$\sigma u f s$ & $\begin{array}{l}\text { T urti } \\
\text { Imm }\end{array}$ & Adultes & $\begin{array}{l}\mathrm{P} p e \\
\mathrm{Imm}\end{array}$ & $\begin{array}{l}\text { similis } \\
\text { Adultes }\end{array}$ & $\begin{array}{c}\text { A sp } \\
\text { Adultes }\end{array}$ & $\begin{array}{l}\text { Préda- } \\
\text { teurs } \\
\text { OEufs }\end{array}$ \\
\hline m 4 & 10,2 & 3,7 & 0,8 & 2,6 & 0,4 & 0,7 \\
\hline s 5,5 & 15,5 & 3,4 & 0,9 & 2,2 & 0,8 & 0,9 \\
\hline
\end{tabular}

et Vedettos, les différences moyennes entre les compartiments $A$ et $B$ ont atteint au maximum $226 \mathrm{~g}$ en poids frais de tomates par plant en faveur du compartiment A. Par contre, pour Fernova, cette observation s'inverse et un excédent moyen de $194 \mathrm{~g}$ par plant est observé pour la lutte biologique (tableau II). Aucune différence significative n'apparaît en ce qui concerne le calibre des fruits $(P>0,05)$. Il convient néanmoins de noter que la récolte des fruits de gros calibre fut importante et plus précoce dans le compartiment biologique (tableau III). En d'autres termes, il apparaît qu'un plant de tomate peut supporter des dégâts relativement importants au niveau foliaire avant que la production de fruits ne soit affectée. Selon Stacey (1983), une perte effective de $25 \%$ de la surface foliaire des plants de to-

Tableau II. Rendements d'une culture de tomates en serre en fonction du cultivar et du type de lutte appliquée contre l'acarien tisserand. Ces rendements sont calculés pour 20 plants par cultivar. La rubrique calibre représente le nombre moyen de fruits par plant de ce calibre.

\begin{tabular}{|c|c|c|c|c|c|c|}
\hline \multirow[t]{2}{*}{ Cultivar } & \multirow[t]{2}{*}{ Lutte } & \multirow{2}{*}{$\begin{array}{l}\text { Rendement } \\
\text { (kg/plant) }\end{array}$} & & \multicolumn{3}{|c|}{ Calibre $(\mathrm{mm})$} \\
\hline & & & & $<57$ & $57-67$ & $>67$ \\
\hline \multirow[t]{2}{*}{ Caruso } & Classique & 8,311 & $\begin{array}{l}\text { Nombre } \\
\text { Pourcentage }\end{array}$ & $\begin{array}{l}2,5 \\
6,0\end{array}$ & $\begin{array}{r}5,1 \\
12,1\end{array}$ & $\begin{array}{l}34,4 \\
81,9\end{array}$ \\
\hline & Biologique & 8,292 & $\begin{array}{l}\text { Nombre } \\
\text { Pourcentage }\end{array}$ & $\begin{array}{l}1,3 \\
3,4\end{array}$ & $\begin{array}{l}3,0 \\
7,9\end{array}$ & $\begin{array}{l}33,9 \\
88,7\end{array}$ \\
\hline \multirow[t]{2}{*}{ Vedettos } & Classique & 6,529 & $\begin{array}{l}\text { Nombre } \\
\text { Pourcentage }\end{array}$ & $\begin{array}{l}3,7 \\
8,5\end{array}$ & $\begin{array}{l}10,2 \\
23,5\end{array}$ & $\begin{array}{l}29,5 \\
68,0\end{array}$ \\
\hline & Biologique & 6,303 & $\begin{array}{l}\text { Nombre } \\
\text { Pourcentage }\end{array}$ & $\begin{array}{r}4,1 \\
10,0\end{array}$ & $\begin{array}{r}8,5 \\
20,7\end{array}$ & $\begin{array}{l}28,4 \\
69,3\end{array}$ \\
\hline \multirow[t]{2}{*}{ Fernova } & Classique & 6,474 & $\begin{array}{l}\text { Nombre } \\
\text { Pourcentage }\end{array}$ & $\begin{array}{l}1,8 \\
4,6\end{array}$ & $\begin{array}{r}4,5 \\
11,4\end{array}$ & $\begin{array}{l}33,2 \\
84,1\end{array}$ \\
\hline & Biologique & 6,668 & $\begin{array}{l}\text { Nombre } \\
\text { Pourcentage }\end{array}$ & $\begin{array}{l}1,1 \\
2,8\end{array}$ & $\begin{array}{r}4,2 \\
10,6\end{array}$ & $\begin{array}{l}34,3 \\
86,6\end{array}$ \\
\hline
\end{tabular}


mates causée par des piqûres d'acariens phytophages peut être tolérée sans perte de rendement. Le même auteur montre que malgré une défoliation artificielle des $3 / 4$ de la surface de chaque feuille, le plant de tomate est encore capable de produire $60 \%$ du rendement d'un plant entier. II y a donc un mécanisme de compensation probablement lié à une augmentation de l'activité photosynthétique de la surface restante.

Actuellement une étude de l'impact des dégâts d'acariens sur l'activité photosynthétique et sur la résistance stomatique des feuilles de plants de tomates est en cours (Hance et al, 1990).

\section{Comparaison des coûts}

Pour la lutte biologique, les coûts totalisés des différentes interventions s'élèvent à $2223 \mathrm{~F}$ belges pour $100 \mathrm{~m}^{2}$ de culture (tableau IV). Ce montant est ventilé comme suit : $536 \mathrm{FB}$ pour les lâchers de $P$ persimilis, 446 FB pour l'introduction d'E formosa, $665 \mathrm{FB}$ pour la lutte avec $\mathrm{N}$ cucumeris, et 476 FB pour les lâchers de $D$ sibirica et du $D$ isaea; d'autre part, les frais relatifs à l'introduction d'A andersoni (élevé au laboratoire) et des pièges jaunes placés en début de culture s'élèvent à environ $100 \mathrm{FB}$, l'introduction préalable de l'acarien tisserand étant pratiquement négligeable. Au cours de la même période, le coût des insecticides et acaricides utilisés pour la lutte chimique s'est élevé à $2150 \mathrm{FB} / 100 \mathrm{~m}^{2}$. La protection de la culture a donc nécessité un investissement équivalent pour les 2 techniques vitilisées.

Les revenus par cultivar et par type de lutte dépendent fortement de la fluctuation quotidienne des prix des différents calibres qui ont varié jusqu'à $17 \mathrm{FB} / \mathrm{kg}$ en $2 \mathrm{j}$ (tableau III). Ce facteur de type économique mais peu prévisible est rarement pris en compte dans les recherches relatives à la lutte intégrée. Pourtant, il conditionne les revenus et peut avoir un impact important dont il faut tenir compte. En effet, bien que les différences de rendements et de calibrations ne soient pas statistiquement significatives, un test de $\chi^{2}$ appliqué sur les revenus nets montre des différences significatives entre la lutte chimique et la lutte biologique $(P \geq 0,01)$. Ceci s'explique par la combinaison de 3 facteurs :

- les rendements plus élevés à la suite de la lutte biologique pour le cultivar Fernova,

- la proportion plus grande de fruits de gros calibre pour la lutte biologique,

- la valeur élevée des fruits de gros calibre au début de la récolte (tableau II). Tous cultivars confondus, on observe un excédent de revenu de $973 \mathrm{FB}$ par $100 \mathrm{~m}^{2}$ pour la lutte biologique.

Tableau III. Évolution des prix par calibre et des nombres moyens de fruits de chaque calibre produits par plant pour une culture de tomates en serre.

\begin{tabular}{|c|c|c|c|c|c|c|c|c|c|c|}
\hline \multirow[t]{3}{*}{ Cultivar } & \multirow[t]{3}{*}{ Lutte } & \multicolumn{9}{|c|}{ Calibre } \\
\hline & & \multicolumn{3}{|c|}{$<57$} & \multicolumn{3}{|c|}{ [57-67] } & \multicolumn{3}{|c|}{$>67$} \\
\hline & & $1^{*}$ & 2 & 3 & 1 & 2 & 3 & 1 & 2 & 3 \\
\hline \multirow[t]{2}{*}{ Caruso } & Classique & - & 0,2 & 2,3 & - & 0,8 & 4,3 & 0,4 & 20,2 & 14,0 \\
\hline & Biologique & - & - & 1,3 & 0,1 & 0,6 & 2,3 & 0,7 & 20,9 & 12,3 \\
\hline \multirow{2}{*}{ Vedettos } & Classique & - & 0,5 & 3,2 & 0,1 & 2,9 & 7,2 & 0,8 & 19,6 & 9,1 \\
\hline & Biologique & - & 0,1 & 4,0 & 0,2 & 2,5 & 5,8 & 2,3 & 20,6 & 5,5 \\
\hline \multirow{2}{*}{ Fernova } & Classique & 0,1 & 0,3 & 14,0 & - & 0,7 & 3,8 & 0,9 & 17,4 & 15,0 \\
\hline & Biologique & - & - & 1,1 & - & 0,7 & 3,5 & 2,2 & 16,5 & 16,0 \\
\hline $\begin{array}{l}\text { Prix moyen } \\
\text { pendant la période }\end{array}$ & & 19,7 & 18,1 & 15,0 & 18,7 & 20,1 & 17,0 & 19,5 & 20,2 & 17,0 \\
\hline
\end{tabular}

* 1 : période du 21 au 28 juin; 2 : période du 30 juin au 28 juillet; 3 : période du 31 juillet au 22 août. 
Tableau IV. Revenus et coûts comparatifs des deux types de protection exprimés en francs belges (FB) par $100 \mathrm{~m}^{2}$ de culture de tomates. ${ }^{\star \star}$ Différence significative entre les traitements.

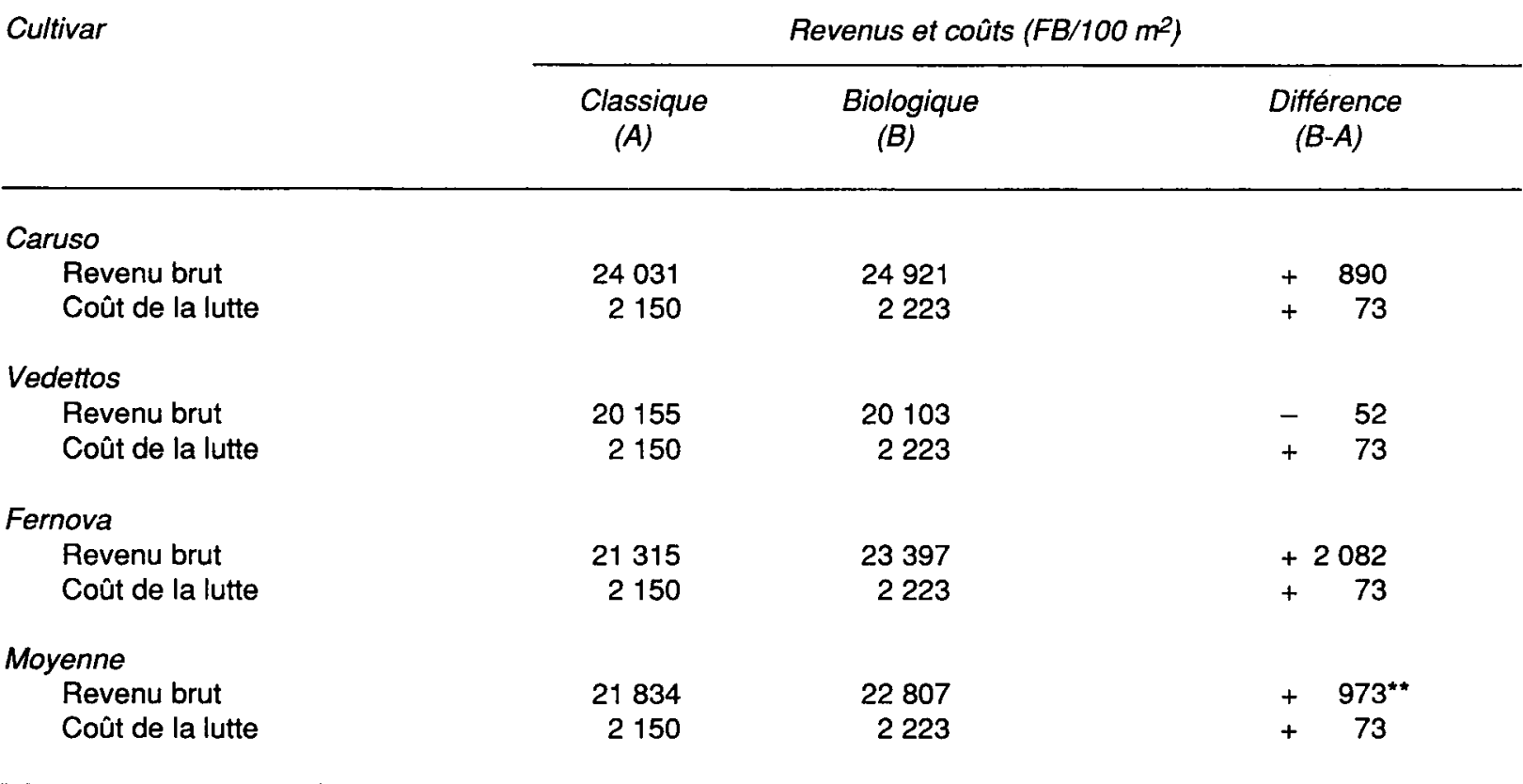

\section{DISCUSSION ET CONCLUSIONS}

L'application de la technique de l'introduction préalable du ravageur (IPR) rencontre un obstacle d'ordre psychologique qu'il convient de mentionner. En effet, introduire volontairement le ravageur que l'on combat peut, à première vue, paraître risqué pour l'horticulteur. Cette technique représente pourtant un moyen de lutte dont les effets peuvent même se faire sentir au niveau des cultures suivantes. C'est en effet au cours de la première année que les problèmes s'avèrent les plus importants, et que l'équilibre est le plus difficile à atteindre. Par la suite, l'installation d'une population naturelle de prédateurs dans la serre, alliée au recours à d'autres techniques (comme le contrôle de la diapause du phytophage, par exemple), facilite la lutte contre le ravageur (Hussey et Scopes, 1985).

II est apparu que la densité de prédateurs était insuffisante en juillet pour faire face à l'invasion massive des tétranyques, et un apport supplémentaire de prédateurs fut nécessaire. II conviendrait donc d'augmenter le nombre d'acariens introduits au départ (à la fois proies et prédateurs), plus particulièrement au niveau des parois de la serre. La prévention des infections pourrait être également complétée par exemple en plaçant une toile à fines mailles sur les ouvertures d'aé- ration, ou encore en inhibant l'entrée en diapause de l'acarien phytophage par le recours aux flashes lumineux lors des périodes sensibles (Barker et al, 1964) pour éviter les infestations précoces de printemps. L'utilisation de cultivars de tomates potentiellement moins sensibles au phytophage devrait être également encouragée, d'autant qu'un test rapide pour détecter cette résistance intrinsèque a été mis au point (Van Impe, 1988).

Au vu de l'expérience réalisée, il est difficile de scinder les effets respectifs de $P$ persimilis et de $\boldsymbol{A}$ andersoni. En fin d'expérience, leurs niveaux de densité sont différents et confirment la croissance plus rapide des populations de $P$ persimilis. Cette espèce est en effet douée d'un potentiel démographique plus élevé que celui $d^{\prime} A$ andersoni et même que celui de leur proie commune, $T$ urticae (Hance, 1988). Cependant, des expériences similaires réalisées en présence de $P$ persimilis seuls, ont montré que les quantités de prédateurs à introduire sont nettement supérieures aux quantités introduites ici (Nihoul et Van Impe, 1991). Enfin, des expériences réalisées dans de petites enceintes expérimentales ont montré qu'une introduction d'Amblyseius andersoni seul serait insuffisante pour contrecarrer le développement estival des populations d'acariens phytophages (Hance et al, 1991). Néanmoins, avant de proposer des plans de lutte im- 
pliquant les 2 types de prédateurs, il sera nécessaire de répéter ces expériences en serre de production et de porter une attention toute particulière à la répartition spatiale des 2 espèces et éventuellement aux phénomènes de compétitions. À l'avenir, des expériences concernant différentes espèces d'Amblyseius et plusieurs niveaux de densités de départ sont à programmer, afin de déterminer les modalités pratiques d'utilisations de ce type de prédateurs.

En conclusion, bien qu'il soit encore nécessaire d'approfondir la recherche, la lutte biologique en culture de tomates en serres chauffées apparaît pleinement réalisable sans pertes significatives de rendements pondéraux, ni augmentation des coûts de production, mais l'application des techniques de lutte ingérée requiert une technicité plus spécifique dont le développement doit encore être amélioré.

\section{REMERCIEMENTS}

Les auteurs remercient B Capelle et $L$ Renier pour leur aide technique efficace tout au long de la culture. Cette recherche a été financée par l'Institut pour l'encouragement de la recherche scientifique dans l'industrie et l'agriculture (IRSIA). Th Hance a disposé d'un mandat de chargé de recherches du Fond national de la recherche scientifique de Belgique (FNRS). $A$ andersoni a été fourni par le Dr M Baillod de la Station agronomique de Changins (Suisse). Le pollen provient du Centre apicole de recherches et d'informations (CARI) de Louvain-la-Neuve et sa composition a été déterminée par Madame $\mathrm{C}$ Delcourt.

\section{RÉFÉRENCES}

Amano H, Chant DA (1977) Life history and reproduction of two species of predacious mites, Phytoseiulus persimilis Athias-Henriot and Amblyseius andersoni (Chant). (Acarina: Phytoseiidae). Can J Zool 55, 1978-1983

Baillod M, Schmid A, Guignard E, Antonin P, Caccia R (1982) Lutte biologique contre l'acarien rouge en viticulture. II. Équilibres naturels, dynamique des populations et expériences de lâcher de typhlodromes. Rev Suisse Vitic Arboric Hortic 14, 345352

Barker RJ, Cohen CF, Mayer A (1964) Photoflashes, a potential new tool for control of insect populations. Science 145, 1195-1197

Bernstein C (1985) A simulation model for an acarine predator-prey system (Phytoseiulus persimilis - Tetranychus urticae). J Anim Ecol 54, 375-389

Gambaro PI (1983) II controllo biologico degli acari sul melo. I) L'effeto dannoso di alcumi fungicidi sugli acari predatori. Inf Agr 39, 26951-26953
Hance Th (1988) Les paramètres démographiques de Phytoseiulus persimilis Athias-Henriot (Acari:Phytoseiidae) en relation avec ses possibilités de contrôle de Tetranychus urticae Koch (Acari: Tetranychidae). Annu Soc $R$ Zool Belg 118, 161170

Hance T, Pasleau A (1987) Étude de la relation triphasique Haricot-Tetranychus urticae-Phytoseiulus persimilis et de l'impact positif du prédateur sur la plante hôte. Med Fac Landbouww Rijksuniv Gent $52,433-448$

Hance T, Maréchal B, Van Impe G (1990) Réalisation d'une échelle à 5 niveaux pour l'évaluation des dégâts provoqués par le Tetranychus urticae en culture de tomates. Phytoprotection 71, 143-144

Hance T, Pasleau A, Nihoul P (1991) Étude des potentialités de Amblyseius andersoni (Acari: Phytoseiidae) pour le contrôle biologique de l'acarien phytophage Tetranychus urticae- (Acari: Tetranychidae). Med Fac Landbouww Rijksuniv Gent 56 12a, 325-341

Hassan SP, Oomen PA (1985) Testing the side effects of pesticides on beneficial organisms by OILB working party. In: Biological pest control. The glasshouse experience (NW Hussey and NEA Scopes, eds). Blandford Press, Poole, UK, 145-151

Hussey NW, Scopes NEA (1985) Mite management for greenhouse vegetables in Britain. In: Spider Mites. Their biology, natural enemies and control (W Helle, MW Sabelis, eds) Elsevier, Amsterdam, 285-297

Maréchal B (1990) Red spider mite assessment for greenhouse tomatoes. Belg J Zool 120, 79

Nihoul P, Van Impe G (1991) Contrôle intégré de Tetranychus urticae en culture de tomates. Med Fac Landbouww Rijksuniv Gent 56, 12a, 319-324

Rambier A, van de Vrie M (1974) Les acariens auxilliaires prédateurs de tétranyques dans les vergers de pommiers. In: Les organismes auxiliaires en vergers de pommiers OLL, Wageningen, Pays-Bas 211-214

Putman WL, Herne DHC (1966) The role of predators ans other biotic agents in regulating the population density of phytophagous mites in Ontario peach orchards. Can Entomol 98, 808-820

Stacey DL (1983) The effect of artificial defoliation on the yield of tomato plants and its relevance to pest damage. J Hortic Sci 58, 117-120

Van Impe G (1985) Contribution à la conception de stratégies de contrôle de l'Acarien tisserand commun, Tetranychus urticae Koch (Acari: Tetranychidae). Dissertation doctorale, Université Catholique de Louvain, Louvain-la-Neuve, $382 p$

Van Impe G (1988) Peut-on évaluer la résistance d'une plante aux tétranyques? Agra-magazine 6, 43-44

Van Rijn PCJ, Sabelis MW (1990) Pollen availability and its effect on the maintenance of populations of Amblyseius cucumeris, a predator of thrips. Med Fac Landbouww Rijksuniv Gent 55, 335-341 